\title{
Task-related functional connectivity of the caudate mediates the association between trait mindfulness and implicit learning in older adults
}

\author{
Chelsea M. Stillman ${ }^{1} \cdot{\text { Xiaozhen } \text { You }^{2} \cdot \text { Kendra L. Seaman }}^{3} \cdot$ Chandan J. Vaidya $^{4}$. \\ James H. Howard Jr. ${ }^{5}$. Darlene V. Howard ${ }^{4}$
}

Published online: 27 April 2016

(C) Psychonomic Society, Inc. 2016

\begin{abstract}
Accumulating evidence shows a positive relationship between mindfulness and explicit cognitive functioning, i.e., that which occurs with conscious intent and awareness. However, recent evidence suggests that there may be a negative relationship between mindfulness and implicit types of learning, or those that occur without conscious awareness or intent. Here we examined the neural mechanisms underlying the recently reported negative relationship between dispositional mindfulness and implicit probabilistic sequence learning in both younger and older adults. We tested the hypothesis that the relationship is mediated by communication, or functional connectivity, of brain regions once traditionally considered to be central to dissociable learning systems: the caudate, medial temporal lobe (MTL), and prefrontal cortex (PFC). We first replicated the negative relationship between mindfulness and implicit learning in a sample of healthy older adults (60-90 years old) who completed three event-related
\end{abstract}

Electronic supplementary material The online version of this article (doi:10.3758/s13415-016-0427-2) contains supplementary material, which is available to authorized users.

Chelsea M. Stillman

cmstillm@gmail.com

1 Department of Psychiatry, University of Pittsburgh Medical Center, 4805 Sennott Square, 203 S Bouquet Street, Pittsburgh, PA, USA

2 Department of Psychology, Children's National Medical Center, Washington, DC, USA

3 Department of Psychology, Yale University, New Haven, CT, USA

4 Department of Psychology, Georgetown University, Washington, DC, USA

5 Department of Psychology, The Catholic University of America, Washington, DC, USA runs of an implicit sequence learning task. Then, using a seedbased connectivity approach, we identified task-related connectivity associated with individual differences in both learning and mindfulness. The main finding was that caudate-MTL connectivity (bilaterally) was positively correlated with learning and negatively correlated with mindfulness. Further, the strength of task-related connectivity between these regions mediated the negative relationship between mindfulness and learning. This pattern of results was limited to the older adults. Thus, at least in healthy older adults, the functional communication between two interactive learning-relevant systems can account for the relationship between mindfulness and implicit probabilistic sequence learning.

Keywords Implicit learning $\cdot$ Mindfulness $\cdot$ Functional connectivity

\section{Introduction}

Mindfulness refers to the ability to stay attentive and receptive to events and experiences taking place in the present (Brown \& Ryan, 2003). However, in the mindfulness literature there are distinctly different uses of the term "mindfulness." For example, dispositional mindfulness refers to naturally occurring variation among individuals in their propensity for mindfulness. In contrast, mindfulness can also be cultivated through training. The present study focuses on dispositional mindfulness. As a general construct, mindfulness has gained popularity in recent years because mindfulness training is associated with a number of positive emotional and cognitive health outcomes. For example, it improves explicit cognitive functioning, or that which occurs with conscious intent and awareness (e.g., Jha, Stanley, Kiyonaga, Wong, \& Gelfand, 2010; Mrazek, Franklin, Phillips, Baird, \& Schooler, 2013; 
Mrazek, Smallwood, \& Schooler, 2012; Schmertz, Anderson, $\&$ Robins, 2009). However, two recent studies have reported a negative relationship between dispositional mindfulness and implicit types of learning and memory, or those that occur without conscious awareness or intent (Stillman, Feldman, Wambach, Howard, \& Howard, 2014; Whitmarsh, Uddén, Barendregt, \& Petersson, 2013). This apparent dissociation between implicit and explicit processes raises the possibility that mindfulness is not beneficial to all cognitive domains.

The negative relationship between dispositional mindfulness and implicit probabilistic sequence learning (IPSL) reported in Stillman et al. (2014), and the potential neural mechanisms behind it, are the focus of the present functional magnetic resonance (fMRI) study. In particular, we examine whether the relationship is mediated by communication, or functional connectivity, among three brain regions: the caudate, prefrontal cortex (PFC), and medial temporal lobe (MTL). At the core of our hypothesis regarding these regions is the fact that the PFC and MTL were once considered central regions of a distinct learning system which competes with the "implicit" learning system supported by the caudate for control of behavior (Squire, 2004). However, recent views have challenged this simplistic process-pure distinction, positing that the MTL and PFC are also involved in aspects of implicit processes (e.g., Henke, 2010). Thus, we hypothesized that the relative balance of the MTL and caudate-based learning systems (perhaps fostered by their functional connectivity) is the neural mechanism by which mindfulness is negatively associated with IPSL.

\section{Functional connectivity and implicit learning}

Caudate-MTL connectivity There is evidence that the balance between these interacting learning systems is important for IPSL. The caudate's involvement in this type of learning is well established (Gamble et al., 2014; Rieckmann, Fischer, \& Bäckman, 2010; Simon, Stollstorff, et al., 2011; Simon, Vaidya, Howard, \& Howard, 2012). However, there is also evidence that the MTL, a region once thought to exclusively support functions of the explicit learning system, supports components of IPSL as well. For example, studies have shown that the MTL is coactivated with the caudate during IPSL tasks, and that a specific temporal pattern of activation in these regions is associated with learning outcomes (Albouy et al., 2008; Gheysen, Van Opstal, Roggeman, Van Waelvelde, \& Fias, 2011; Poldrack et al., 2001; Rieckmann et al., 2010; Rose, Haider, Weiller, \& Büchel, 2002; Schendan, Searl, Melrose, \& Stern, 2003; Schendan, Tinaz, Maher, \& Stern, 2013; Simon et al., 2012). In addition, the integrity of white matter tracts connecting both the MTL and caudate to the dorsolateral PFC has been shown to positively relate to IPSL (Bennett, Madden, Vaidya, Howard, \& Howard, 2011). These seemingly paradoxical findings suggest that more communication/coordination between regions belonging to two interacting learning systems is beneficial for IPSL.

A recent study of resting state functional connectivity further supports this idea in that it provides the first direct evidence that functional communication between the caudate and MTL is related to IPSL aptitude (Stillman et al., 2013). Resting state functional connectivity refers to the measurement of spontaneous neural activity in a task-free state, and so is thought to reveal the intrinsic architecture of the brain (Raichle, 2010). Individuals with stronger, more positive intrinsic functional connectivity between the caudate and right MTL learned more on a subsequent IPSL task, suggesting that perhaps positive connectivity between these regions prior to learning enables them to communicate and interact more efficiently during learning (i.e., promotes "readiness to learn"). However, this hypothesis could not be tested directly because no fMRI data were collected while participants were performing the IPSL task. Regardless of this limitation, these findings add to the fMRI and diffusion tensor imaging (DTI) evidence highlighting the importance of communication between the caudate and MTL for IPSL.

Caudate-PFC connectivity There is also evidence, albeit more tentative, that communication between the caudate and PFC is relevant to IPSL. One DTI study has shown that the integrity of white matter tracts between the caudate and dorsolateral PFC is positively related to learning, suggesting that greater communication between these regions benefits IPSL (Bennett et al., 2011). In contrast, behavioral studies show that IPSL improves when the involvement of frontal brain regions is reduced. For example, IPSL improves following inhibitory theta burst stimulation to the dorsolateral PFC, as well as following hypnosis, a procedure thought to disrupt the functional connections of prefrontal brain regions underlying cognitive control (Galea, Albert, Ditye, \& Miall, 2009; Nemeth, Janacsek, Polner, \& Kovacs, 2012). While none of these studies measured functional connectivity between the caudate and PFC directly, one potential interpretation of these results is that less regulation of the caudate by prefrontal regions (and perhaps less functional connectivity between them) benefits IPSL.

\section{Functional connectivity and mindfulness}

In addition to its importance for IPSL, communication between the two learning systems may also be related to mindfulness.

Caudate-PFC connectivity Several lines of evidence, for example, suggest connectivity between the caudate and PFC is relevant for mindfulness. However, there are two opposing views regarding how connectivity between these regions should relate to mindfulness. The "regulatory" 
account suggests that mindfulness might increase the PFC's ability to exert top-down regulation of subcortical systems (e.g., Chiesa, Serretti, \& Jakobsen, 2013), and thus predicts that mindfulness may increase PFC connectivity to subcortical regions, thereby promoting top-down regulation of functions that are otherwise habitual. In contrast, the "reduced reactivity" account posits that mindfulness might reduce the need for such top-down regulation (e.g., Shapiro, Carlson, Astin, \& Freedman, 2006), raising the possibility that mindfulness may be associated with less connectivity between the caudate and PFC. The majority of evidence favors the regulatory account (Garland, Froeliger, \& Howard, 2014; Grant, Courtemanche, Duerden, Duncan, \& Rainville, 2010; Luders, Toga, Lepore, \& Gaser, 2009; Tang et al., 2009, 2010; Tang, Tang, \& Posner, 2013), but some recent evidence is consistent with the reduced reactivity account (Kirk, Brown, \& Downar, 2014; Kirk \& Montague, 2015; Taren, Creswell, \& Gianaros, 2013; Westbrook et al., 2013). Thus, while caudate-PFC connectivity might be related to mindfulness, it is ultimately hard to predict the direction of this relationship based on the mixed (and predominately indirect) nature of the existing evidence.

Caudate-MTL connectivity It is similarly difficult to predict how caudate-MTL connectivity - another link between the two learning systems - might relate to mindfulness, as few studies of mindfulness have focused on these regions. There is evidence, however, that the structure and functioning of the caudate and MTL change following mindfulness training (Holzel et al., 2011; Kirk \& Montague, 2015; Lutz et al., 2014; Pickut et al., 2013; Tang et al., 2009; Zeidan et al., 2011). The structure of both of these regions has also been shown to negatively relate to dispositional mindfulness (Taren et al., 2013). This evidence, coupled with the fact that caudateMTL connectivity is important to IPSL, led us to reason that caudate-MTL connectivity might be a mechanism by which the balance of these learning systems is modulated.

\section{Present study}

The present study has three main aims. The first aim is to replicate the negative relationship between dispositional mindfulness and IPSL reported in Stillman et al. (2014). The second aim is to identify functional connections during the task related to individual differences in learning, thus extending the results reported in an earlier resting state study of the same type of learning (Stillman et al., 2013). Finally, a third aim is to assess whether either of the functional connections between learning systems discussed above (caudate-PFC or caudate-MTL) mediates the relationship between mindfulness and IPSL.
Participants completed a self-report questionnaire assessing their level of dispositional mindfulness. Then, on a later day, they completed three runs of an IPSL task while undergoing MRI. Based on the results of our previous behavioral study (Stillman et al., 2014), we predicted that there would be a negative relationship between mindfulness and IPSL. Based on the results of Stillman et al. (2013) and the broader IPSL literature, we predicted that caudate-MTL connectivity would be positively correlated with learning, but that caudate-PFC connectivity might be negatively correlated with learning. Based on indirect evidence from the mindfulness literature, we predicted that caudate-PFC and caudate-MTL connectivity would be correlated with mindfulness. However, we were not able to make a clear prediction regarding the direction of these relationships, as the existing evidence implicating these connections is mixed and comes predominately from studies of mindfulness training.

\section{Materials and methods}

\section{Participants}

Fifty younger adults aged 18-37 years and 51 community-dwelling older adults aged 60-90 years were recruited from Georgetown University or the surrounding Washington, DC area to participate in a multi-day study. Most of these participants ( $\mathrm{N}=45$ younger; $\mathrm{N}=47$ older) completed an fMRI-scanning day. Exclusion criteria included self-reported use of (1) psychotropic medications (e.g., stimulants), (2) neurological injury, disease or psychiatric diagnosis, (3) contraindications for MRI (e.g., presence of irremovable metal in the body), and (4) left-handedness. Three younger and five older participants were excluded from the final imaging sample due to technical difficulties during scanning $(\mathrm{N}=3)$, inability to complete the scan $(\mathrm{N}=2)$, or because it was subsequently determined that they met study exclusion criteria $(\mathrm{N}=3)$. The final sample for the present study therefore consisted of 42 adults in each age group (younger adult mean $\pm \mathrm{SD}$ age $=22.0 \pm 3.8,29$ female; older adult mean \pm SD age $=70.4 \pm 6.1,19$ female). Neuropsychological functioning was assessed on a behavioral testing day prior to the scan; all participants were deemed cognitively healthy (Table 1).

All participants gave informed consent in accordance with the guidelines of the Declaration of Helsinki and the Georgetown University Institutional Review Board. They were compensated with a US\$50 gift card for their participation in the fMRI component of the study and US\$25 for completing the behavioral components. 
Table 1 Participant demographics and neuropsychological test results

\begin{tabular}{|c|c|c|}
\hline Measure & $\begin{array}{l}\text { Younger adults } \\
\text { Mean (SD) }\end{array}$ & $\begin{array}{l}\text { Older adults } \\
\text { Mean (SD) }\end{array}$ \\
\hline Age & $22.0(3.7)$ & $70.4(6.1)$ \\
\hline Education & $14.3(1.9)$ & $18.0(3.0)$ \\
\hline NAART $^{\mathrm{a}}$ & $15.6(6.4)$ & $8.3(5.7)$ \\
\hline BDS & $7.6(2.2)$ & $6.7(1.9)$ \\
\hline DSST & $90.4(15.1)$ & $66.0(14.1)$ \\
\hline MMSE & - & $29.0(1.0)$ \\
\hline MAAS & $4.2(.82)$ & $4.5(0.68)$ \\
\hline Self-reported health ${ }^{\mathrm{b}}$ & $4.6(.49)$ & $4.5(0.73)$ \\
\hline
\end{tabular}

${ }^{\mathrm{a}}$ For the NAART, higher scores reflect worse performance. For all other measures, higher scores reflect better performance

b Self-reported health scores ranged from 1 (poor) to 5 (Excellent)

$B D S$ Backwards Digit Span, DSST Digit Symbol Substitution Test, MAAS Mindful Attention Awareness Scale, MMSE Mini Mental State Examination, NAART North American Adult Reading Test

\section{fMRI paradigm and image acquisition}

We measured implicit learning in the present study using a sequence learning task called the Triplets Learning Task (TLT). The task was a shorter and slightly modified version from that reported in Howard et al. (2008). As shown in Fig. 1, participants viewed four open circles on a computer screen. The circles were displayed against a light gray background. Each trial, or "triplet", consisted of three events: the sequential presentation of two cue events, each consisting of one of the open circles filling in red, followed by a target event in which one of the circles filled in green. Each cue was displayed for $120 \mathrm{~ms}$ and the green target remained on the screen for 760 $\mathrm{ms}$, with $150 \mathrm{~ms}$ separating each event within a trial. Participants were instructed to observe the first two red events and to respond to the location of the green target as quickly as possible using spatially corresponding buttons held in the left and right hands (two buttons in each hand). Unbeknown to participants, some triplets occurred more often than others; thus there was a probabilistic regularity embedded in the task.

Forty-eight triplets (out of a possible 64) were presented (Appendix Table 2). A set of 16 triplets, referred to as Trills (e.g., 121, 232) and Reps (e.g., 111, 222), were not presented during the TLT task because they have been shown to have preexisting response tendencies (D.V. Howard et al., 2004; Soetens, Melis, \& Notebaert, 2004). These triplets were, however, presented as unseen foils in a computer-based recognition task completed after the scan, as described below.

During the TLT, participants completed three runs, each run consisting of five blocks of 48 trials, with brief breaks provided between blocks (i.e., 240 trials in each run, and thus 720 trials total in the TLT). On half the trials in each block, one of eight high probability (HP) triplets occurred (i.e., each HP triplet occurred on $6.25 \%$ of trials); on the other half of trials one of 40 low probability (LP) triplets occurred (i.e., each LP triplet occurred on $1.25 \%$ of trials). The set of HP and LP triplets was counterbalanced across participants (six possible patterns), and all cues and targets occurred an equal number of times. This created an 80:20 ratio of HP versus LP triplets as in the original version of the task, but, unlike the original task, resulted in an equal number of trials in the HP versus LP conditions. Thus, each run was composed of $120 \mathrm{HP}$ and $120 \mathrm{LP}$ trials presented in a rapid, event-related design with a temporally jittered inter-trial interval ranging from 1 to $3 \mathrm{~s}$. Trial and jitter order was presented in the same fixed, pseudorandomized sequence for all participants, optimized using the AFNI make_random_timing tool (Cox, 1996). Given that the present study focused on individual differences, we opted to present HP and LP trials in the same order for everyone in order to ensure that any differences in learning amongst people could not be attributed to differences in the difficulty of various trial sequences.

The TLT was performed in a Siemen's Trio 3-T scanner (Erlangen, Germany). A technician positioned participants in the supine position with a circularly polarized head coil, and fitted padding was inserted to minimize head movements. A mirror mounted on the head coil allowed participants to view task stimuli during scanning.

Functional data were acquired along the AC-PC line using T2*-sensitive gradient Echo Planar Imaging pulse sequence with the following parameters: TR/TE $=2,500 \mathrm{~ms} / 30 \mathrm{~ms}$, $192 \mathrm{~mm} \times 192 \mathrm{~mm}$ field of view (FOV), $64 \mathrm{~mm} \times 64 \mathrm{~mm}$ acquisition matrix, and a $90^{\circ}$ flip angle for an effective resolution of $3 \times 3 \times 3 \mathrm{~mm}$. Forty-seven contiguous 3 -mm thick axial slices were acquired interleaved in the transverse plane. We acquired three event-related runs of the TLT, with 368 volumes per run.

\section{Additional behavioral testing}

The Mindful Attention Awareness Scale (MAAS) On the behavioral test day occurring 1-3 days prior to the scan, participants completed the Mindful Attention Awareness Scale (MAAS; Brown \& Ryan, 2003) to assess their dispositional mindfulness, as well as the battery of neuropsychological tests and questionnaires mentioned above. The MAAS is a singlefactor, self-report questionnaire designed to assess one's tendency to focus on experiences taking place in the present and to disengage from habitual, automatic modes of functioning. The questionnaire contains 15 items that participants rate on a 6 -point Likert scale $(1=$ almost always to $6=$ almost never $)$ how often they experience each item on a day-to-day basis (e.g., "I break or spill things because of carelessness, not paying attention, or thinking of something else," "I do jobs or tasks automatically, without being aware of what I'm doing," "I find it difficult to stay focused on what's happening in the present "). The questionnaire is scored by averaging the participant's 


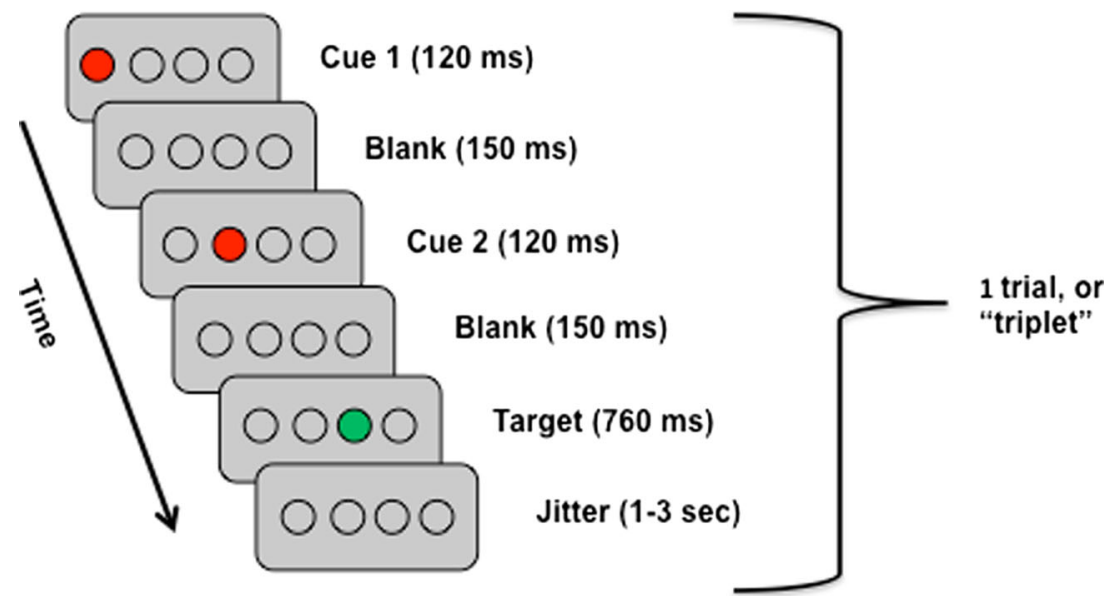

Fig. 1 The Triplets Learning Task (TLT). On each trial, participants observe the first two red cues and then respond to the location of the green target. These discrete, three-event sequences are called "triplets."

responses across all items, with higher scores indicating higher mindfulness. The MAAS has been validated using a variety of subject populations, including healthy younger and older adults (Brown \& Ryan, 2003; Shaurya Prakash, De Leon, Klatt, Malarkey, \& Patterson, 2012), and has been shown to have good psychometric properties (Brown \& Ryan, 2003; Carlson \& Brown, 2005).

Practice TLT Just prior to the scan, participants completed an abbreviated practice run of the TLT in a mock scanner within the imaging facility to ensure that they were comfortable in a scanning environment and understood the task instructions. The practice task was identical to the version of the TLT described above, except that there was no probabilistic regularity that could be learned (i.e., the 48 triplets that would occur later in the TLT were presented at random) and participants completed only 96 trials.

Computer-based recognition task and interview Following the scan, participants completed two sensitive measures to assess their explicit awareness of the probabilistic regularities in the TLT. Computer-based recognition was the main assessment of explicit awareness and so was administered first. During the computer-based recognition task, participants were shown all 48 triplets that occurred during the scan, as well as the remaining possible 16 triplets that had never occurred (as described above); after each, participants indicated via keyboard button press whether they thought that triplet had occurred frequently ("2") or infrequently ("1"). Participants observed the entire triplet before making a frequency rating during the recognition task. We did not have the participants respond to the stimuli during this recognition phase, because we did not want them to be able to base their ratings on feelings of familiarity or response fluency, which could involve implicit
Unbeknown to participants, certain triplets occur with high frequency and others with low frequency in the task

knowledge. The task was therefore designed to probe for explicit/declarative knowledge of the triplet frequencies while minimizing implicit contamination (for discussion see Cohen \& Curran, 1993; Perruchet \& Amorim, 1992; Perruchet \& Gallego, 1993; Willingham, Greeley, \& Bardone, 1993).

After completing the computer-based recognition task, participants were asked a series of increasingly leading questions to probe for strategy use and explicit knowledge. Questions ranged from general (e.g., "Do you have anything to report regarding the task?") to highly specific (e.g., "In fact, there were some regularities or "triplets" that occurred more often than others. Can you name them?).

\section{Image analysis}

Preprocessing The first two images of each functional task run were discarded prior to preprocessing, as they had been included for signal stabilization. Preprocessing was conducted using SPM8 (Wellcome Department of Neurology, London, UK) implemented in Matlab (Version 2013a Mathworks, Inc., Sherborn, MA, USA). Images were corrected for slice timing and motion, normalized to an EPI template, and resliced to a $3 \mathrm{~mm}^{3}$. The images were then smoothed using a Gaussian kernel with full width at half maximum of $8 \mathrm{~mm}$, and temporally filtered with a highpass filter $>.01 \mathrm{~Hz}$.

In the first level analysis we included time series from white matter and CSF segmentations of the MPRAGE image (Van-Dijk et al., 2010) and six motion parameters as regressors of no interest to account for motion and physiological noise. To further control for the spurious effects of motion, volumes exceeding a threshold of $>.5 \mathrm{~mm}$ framewise displacement were deweighted prior to the calculation of connectivity values (Power, Barnes, Snyder, Schlaggar, \& Petersen, 
2012). The global signal was not included as a nuisance signal since recent work has suggested that this procedure may reduce the accuracy of connectivity estimates (Saad et al., 2012).

Seed region-of-interest-creation A bilateral caudate seed region of interest (ROI) including head of the caudate was created using the anatomical AAL atlas included within the Wake Forest University (WFU) pickatlas toolbox implemented in Matlab2013a (Maldjian, Laurienti, \& Burdette, 2004; Maldjian, Laurienti, Kraft, \& Burdette, 2003; TzourioMazoyer et al., 2002). The caudate was chosen as the seed given its well established role in implicit sequence learning in older and younger adults (Aizenstein et al., 2006; Rieckmann et al., 2010; Schendan et al., 2013; Simon, Stollstorff, et al., 2011; Simon et al., 2012), as well as our a priori hypotheses regarding the relevance of functional connectivity between the caudate and medial temporal/prefrontal cortex to individual differences in learning.

Functional connectivity calculation For each participant and for each run, partial correlations were conducted between the bilateral caudate seed time series and that of every other voxel in the brain, while partialling out motion and physiological noise time courses. The resulting $r$ values were converted to $\mathrm{Z}$-scores using Fisher's $\mathrm{r}$ to $\mathrm{z}$ transformation to increase normality of the distribution and allow for further statistical analyses of correlation strengths. This produced a whole brain map of the seed's connectivity during each run of the TLT for each participant.

\section{Primary statistical analyses}

Calculating implicit learning Learning in the TLT was assessed by comparing reaction time (RT) to HP versus LP triplets. Following previous work, we first calculated median RTs for correct responses to each triplet type in each block of 48 trials (e.g., J.H. Howard Jr. et al., 2008; Simon, Howard, \& Howard, 2011). We then averaged these block medians, obtaining a single mean RT for each participant, for each of the two triplet types for each run. A RT difference score (LP minus HP triplet RT) was then computed for each run; thus, higher scores indicate higher learning. Difference scores from the final run (run 3) were used as the learning outcome measure for all analyses reported below, as scores in the final run are thought to represent the cumulative amount learned at the end of exposure to the probabilistic regularity in the task. Throughout the rest of the paper, unless otherwise noted, we use the term learning score to refer to this run 3 difference score between RT on LP minus HP triplets.

Connectivity correlations with implicit learning In order to examine task connectivity of the caudate seed that was correlated with individual differences in learning (aim 2), participants' whole brain connectivity maps were entered as the dependent variable into an ANCOVA model using the GLMFlex toolbox for SPM8 (http://mrtools.mgh.harvard. edu/index.php/GLM Flex). Second-level models created using this toolbox include additional corrections for between-subjects and within-factor error, thus reducing the chances of false positives compared to second-level repeated measures analyses conducted in SPM. Learning scores were included as a covariate of interest, while participants' age and mean task speed were included as covariates of no-interest for all analyses. Results were first assessed at the whole brain level. We then conducted a follow-up analysis in which we repeated the ANCOVA described above, this time restricting the results to specific prefrontal regions using an anatomically defined mask. The frontal mask was created using the AAL atlas within the WFU pickatlas toolbox for SPM and included bilateral oribitofrontal, anterior cingulate, middle frontal, and superior frontal cortices. These regions were chosen because their activity, connectivity, and/or volume have been shown to be modulated by mindfulness training or to co-vary with dispositional mindfulness (for reviews see Tang \& Posner, 2012; Vago \& David, 2012).

Monte Carlo-based correction for multiple comparisons (Ward, 2000) was carried out using tools implemented in Analysis of Functional Imaging (AFNI) software (Cox, 1996). Briefly, we estimated the smoothness of the preprocessed data (3dfwhmx utility), and then conducted 10, 000 simulations of random noise activations with that same smoothness and within the same whole-brain volume as the primary analyses (3DClustSim tool). These simulations revealed that clusters exceeding both a voxelwise alpha of $p<.0005$ and a size of 64 voxels occurred less than $5 \%$ of the time, corresponding to a $\mathrm{p}<.05$ whole brain cluster-level significance threshold. Unless otherwise noted, the results reported below are corrected using this threshold.

\section{Results}

\section{Did participants learn?}

Combined sample Accuracy was very high $(M=.91, S D=$ $.07)$, so few trials were eliminated. Younger adults $(M=.92$, $S D=.05)$ were marginally more accurate than older adults on the task $(M=.90, S D=.09 ; \mathrm{t}(82)=1.69, \mathrm{p}=.06)$.

An independent sample t-test on learning scores (i.e., run 3 difference score between RT on low minus high probability triplets) revealed that younger adults $(M=13.44, S D=14.43)$ learned significantly more than older adults $(M=4.44, S D=$ 20.36 ; $t(82)=2.32, p=.02)$. Learning was negatively correlated with both age, $r(84)=-.25, p=.02$, and education, $\mathrm{r}(84)$ 
$=-.24, \mathrm{p}=.03$. There was also a marginal positive correlation between learning and DSST scores, $\mathrm{r}(84)=.19, \mathrm{p}=.08$.

Younger adults A one-sample t-test revealed that learning scores were significantly greater than zero, $\mathrm{t}(41)=6.04$, $\mathrm{p}<.001$, indicating that younger adults learned as a group. However, there was inter-individual variability in learning within the group (LP-HP difference scores ranged from $-19 \mathrm{~ms}$ to $44.6 \mathrm{~ms})$. Learning scores were correlated with mean task accuracy, $\mathrm{r}(42)=-.44, \mathrm{p}=.004$, and marginally correlated with BDS performance $(\mathrm{r}(42)=.27, \mathrm{p}=.09)$. There were no other correlations with any of the remaining measures reported in Table 1, nor with average speed (ps>.13).

Older adults $A$ one-sample t-test revealed that, on average, learning scores did not differ significantly from zero, $t(41)$ $=1.4, p=.16$, suggesting that there was no learning in the older adult group on average. However, there was substantial inter-individual variability in learning performance (range of LP-HP difference scores $=-32.5 \mathrm{~ms}$ to $44.3 \mathrm{~ms}$ ). Difference scores were not correlated with any of the neuropsychological measures reported in Table 1, nor were they correlated with age, mean task reaction time, or mean task accuracy (all $p \mathrm{~s}$ $>.12$ ), suggesting that individual differences in these measures cannot account for the wide range of scores observed in this group.

\section{Was learning implicit?}

Combined sample Participants' mean frequency ratings on the computer-based recognition task were entered into a mixed effects ANOVA with Age Group (younger, older) as a between-subjects factor and Triplet Type (HP, LP, and unseen foils) as a repeated-measures factor. There was a main effect of triplet type, $F(2,164)=29.00, p<.001$, demonstrating that ratings differed across the three types of triplets presented during the recognition task. Follow-up t-tests revealed that participants rated both HP, $t(83)=5.72, p<.001$, and LP, $t(83)=6.89, p<.0001$, triplets as having occurred more than the unseen foils, and there was no difference in ratings between HP and LP triplets, $t(84)=.92, p=.36$. There was also a marginal Age Group $\times$ Triplet Type interaction, $F(2,164)=$ $2.77, p=.07$. We examined the nature of this interaction by conducting separate ANOVAs on each age group below.

Younger adults A one-way ANOVA on frequency ratings in younger adults revealed a main effect of Triplet Type, $F(2,82)$ $=19.80, p<.0001$. Follow-up t-tests demonstrated that younger adults rated unseen foils significantly lower $(M=1.39, S D$ $=.19)$ than they rated LP $(M=1.58, S D=.13 ; t(41)=6.08, p<$ $.001)$ and HP $(M=1.57, S D=.22 ; t(41)=4.30, p<.001)$ triplets that they had seen during training. However, there was no difference in their ratings to HP and LP triplets, $t(41)$ $=.21, p=.83$.

Older adults A one-way ANOVA on frequency ratings in older adults also revealed a main effect of Triplet Type, $F(2,82)=8.87, \eta_{\mathrm{p}}{ }^{2}=.18, p<.0001$. Follow-up t-tests revealed that, as was also the case for younger adults, older adults rated unseen foils significantly lower $(M=1.46, S D$ $=.25)$ than they rated the $\operatorname{LP}(M=1.54, S D=.22 ; t(41)$ $=3.12, p=.003)$ and HP triplets $(M=1.58, S D=.26 ; t(41)$ $=3.5, p=.001)$, yet there was no difference in their ratings to HP and LP triplets, $t(41)=1.7, p=.11$. The difference in ratings between Seen (HP and LP) versus Unseen (foils) triplets, however, was significantly greater for younger $(M$ $=.19, S D=.19)$ than for older adults, $(M=.09, S D=.17)$; $t(82)=2.50, p=.01)$. This suggests that the interaction reported above was driven by the fact that younger adults were better able to distinguish (in general) which triplets $\mathrm{had} / \mathrm{had}$ not occurred during the TLT task.

This pattern of results suggests that participants in both groups understood the instructions for the recognition task and were complying with them. Critically, however, there was no difference in ratings of the HP versus LP triplets for either group $(p s>.11)$, suggesting that participants could not report which had occurred more often in the task, even though they could reliably distinguish between triplets they saw during training and those they did not.

To further check for explicit awareness at the individual level, we computed recognition difference scores for each participant comparing their mean frequency ratings to LP versus HP triplets, as well as unseen foils versus seen (HP and LP) triplets. Neither score was significantly correlated with our measure of implicit learning, either in the combined group or within each age group ( $p s>14$ ), suggesting that the learning scores are not tapping explicit awareness.

We also conducted a verbal interview for signs of awareness. Of the 42 younger participants, 11 were able to report at least one HP triplet $(\max =3)$, and several reported noticing a pattern in the task. Thus, there was some evidence of awareness from the verbal interview in the younger adults. In contrast, of the 42 older adults, only two were able to name a HP triplet (one reported one HP triplet, and one reported two HP triplets), and only one participant reported noticing a pattern in the task. Beyond this, however, older participants were unable to accurately describe the regularity in the TLT. Overall then, the recognition data suggest that learning in the TLT was largely implicit, particularly for the older group.

\section{Aim 1: Is implicit learning correlated with mindfulness?}

Combined sample Consistent with our prediction (aim 1), as well as the results of Stillman et al. (2014), a correlational analysis revealed that participants' learning scores were 
negatively correlated with their MAAS scores, $r(84)=-.24, p$ $=.03$. This relationship was only marginal in a partial correlation controlling for age and mean task speed, $r(80)=-.19, p=$ .08 . We next examined whether this mindfulness/learning correlation held for each age group separately.

Younger adults There was no significant relationship between mindfulness and learning in the younger adults, $r(38)$ $=-.02, p=.92$. Thus, we failed to replicate the negative relationship found in our earlier studies for this age group.

Older adults Consistent with our hypothesis and earlier study, older adults' learning scores were negatively correlated with their MAAS scores, $r(42)=-.35, p=.02$ (Fig. 2, panel A). The relationship remained significant in a partial correlation controlling for age and mean task speed, $r(38)$ $=-.36, p=.02$.

In addition to examining the relationship between mindfulness and implicit learning, we also tested for a relationship between HP versus LP recognition difference scores (a measure of explicit awareness of the triplet frequencies) and MAAS scores. There was no significant relationship between mindfulness and recognition scores in the combined $(r(80)=$ $-.03, p=.80)$, younger adult $(r(38)=.04, p=.80)$, or older adult sample $(r(38)=-.12, p=.47$; Fig. 2 , panel $\mathrm{B})$, suggesting that the negative relationship between mindfulness and implicit learning on the TLT does not extend to explicit awareness.

Approach for remaining aims The remaining analyses focus only on the older adult sample for several reasons. The main reason is that the critical relationship of interest -that between mindfulness and learning - was significant in the older group, as well as in the combined sample, but it was not significant in the younger group. Thus, the correlation between mindfulness and learning in the combined sample seems to have been driven by the older adults. For this reason, we decided that focusing on the older adults for the remaining aims would most accurately represent the data, a point considered more fully in the discussion. Nonetheless, we did calculate all of the analyses on the combined data from both groups (not reported here), and the pattern of results we report was similar to that reported below for the older adult sample.

An additional reason for focusing only on the older group is that we found some limited evidence of explicit awareness in the younger adults, as evidenced by their questionnaire responses described above, whereas the implicit nature of learning was better established for the older group. Finally, learning in the younger adults was related to mean task accuracy and (marginally) to BDS scores, whereas it was unrelated to any other measures for older adults, which complicated combining the groups.
Aim 2: Does caudate functional connectivity relate to implicit learning success in older adults?

Whole brain results Consistent with our hypothesis, the ANCOVA results in which learning scores and run were covariates of interest, and age and speed were nuisance covariates, revealed an effect of learning in the left (peak MNI x, y, z $=-24,-13,-17 ; \mathrm{k}=177$ ) and right (peak MNI x,y,z $=45$, $-13,-26 ; \mathrm{k}=69)$ medial temporal lobes (MTL). These were the only two clusters to survive whole brain correction, and there were no other significant effects or interactions. The lack of a learning $\times$ run interaction implies that stronger, more positive connectivity between the caudate and MTL during all runs of the TLT is associated with better learning in the final run. This implication was subsequently confirmed with follow-up analyses assessing the correlation between learning and caudate-MTL functional connectivity in each run individually $(r \mathrm{~s}>.41, p \mathrm{~s}<.006)$. Since the relationship was consistent across all runs, the cross-run average connectivity between the caudate and MTL clusters is presented in Fig. 3a and is used for the mediation analyses reported below.

Prefrontal-specific result Contrary to our hypothesis, the whole brain analysis did not reveal any prefrontal clusters whose connectivity to the caudate seed co-varied with individual differences in learning. However, when we conducted the follow-up ANCOVA using a prefrontal mask (because we also had a specific a priori hypothesis about caudate-PFC connectivity) this analysis revealed a cluster in the anterior right middle/superior frontal gyrus (BA10; peak MNI x,y,z = $30,59,19 ; \mathrm{k}=201$ ) whose connectivity with the seed was positively correlated with learning (Fig. 3b). The cluster survived a Monte Carlo-based correction threshold calculated for the masked volume at $p<.05$ (corresponding to an uncorrected threshold $\mathrm{k}=103, p=.005)$.

Additional follow-up analyses When we re-ran the main ANCOVA model reported above using run-specific learning scores, no effects survived correction. Thus, connectivity between the caudate and MTL at all runs was associated with learning scores from the final run, but connectivity during each run was not correlated with the learning scores from that particular run. One possible explanation for this pattern of results is that the strength of connectivity between the caudate and MTL supports learning before the behavioral effects of this learning are observable (at least in some individuals).

Importantly, however, a similar pattern of results to that reported above emerged when we repeated the analyses using fMRI data from Run 3 only, examining its relationship to Run 3 learning scores (Supplementary File 1). Thus, the pattern of results reported in the main model is not driven by the inclusion of fMRI data from all task runs. 

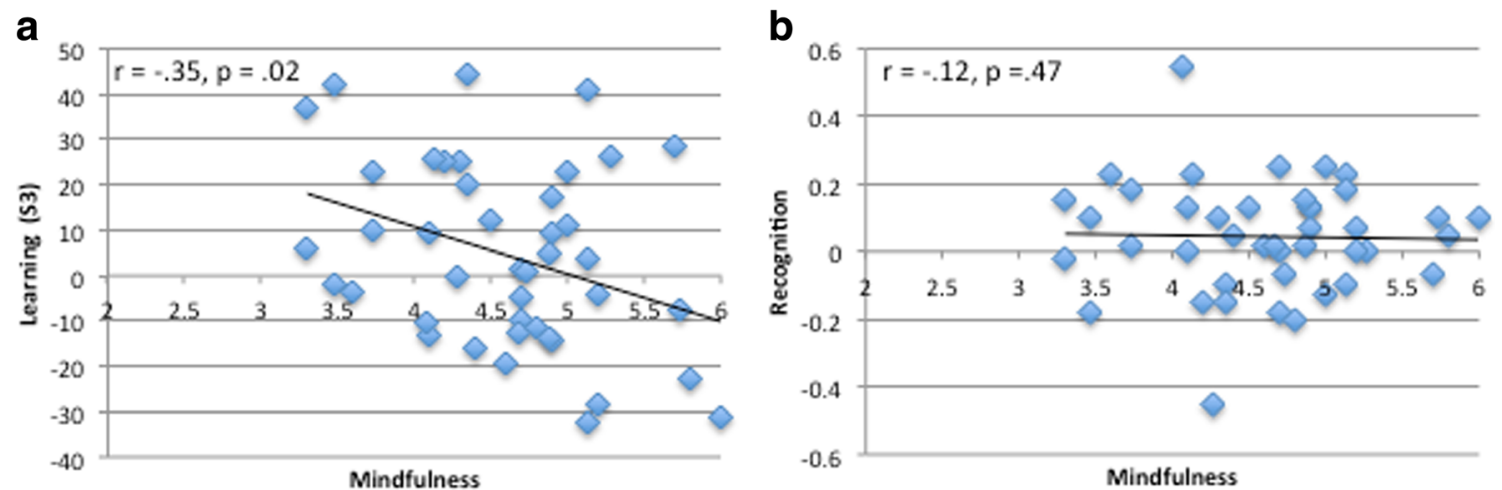

Fig. 2 (a) Self-reported mindfulness is negatively correlated with implicit learning in older adults. (b) Mindfulness is not correlated with recognition scores (a measure of explicit learning)

\section{Do the functional connections related to learning success also relate to mindfulness in older adults?}

Having identified three functional connections associated with learning, we next tested whether the strength of any of these connections was also associated with mindfulness. We found that caudate-lMTL $(r(42)=-.36, p=.02)$ and caudate-rMTL connectivity $(r(42)=-.36, p=.02)$ were negatively correlated with mindfulness (Fig. 4). However, caudate-BA10 connectivity was not correlated with mindfulness $(r(42)=-.19, p=.23)$.

\section{Aim 3: Does functional connectivity mediate the learning-mindfulness relationship in older adults?}

Finally, we tested the two functional connections correlated with both mindfulness and learning (caudate-lMTL, caudaterMTL) as mediators of the relationship between these two behavioral variables (Fig. 5a). We did not examine the caudate-BA10 connection identified as correlating with IPSL above, because it did not correlate with mindfulness scores, indicating it did not qualify as a potential mediator (Baron \& Kenny, 1986). a

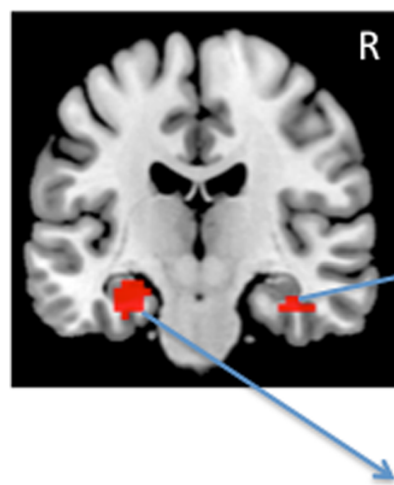

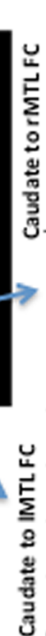
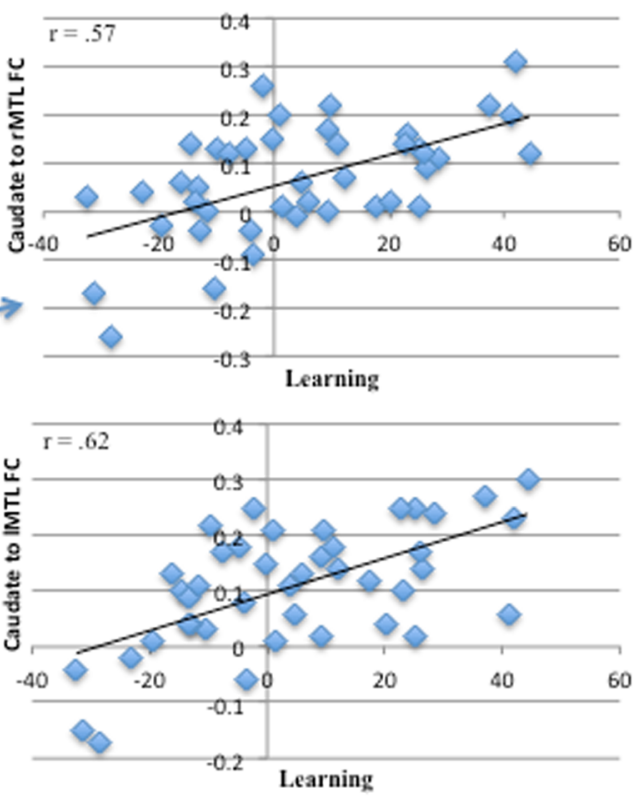

b
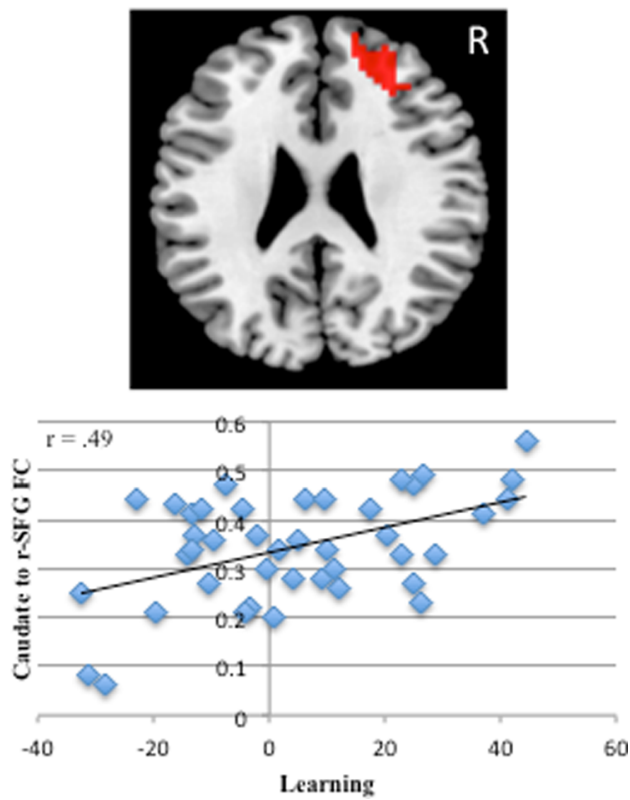

Fig. 3 Task-related functional connectivity of the caudate is associated with individual differences in implicit learning in older adults. (a) Functional connectivity (FC) of the caudate to the left (peak MNI x,y,z $=-24,-13,-17 ; \mathrm{k}=177$ ) and right (peak MNI x,y,z $=45,-13,-17 ; \mathrm{k}$ =69) medial temporal lobe (MTL) was positively associated with learning in the Triplets Learning Task (TLT). The clusters were corrected for multiple comparisons at a whole brain threshold of $\mathrm{P}<.05$. (b)
Functional connectivity of the caudate to the right medial/superior frontal gyrus (BA10; peak MNI x,y,z $=30,59,19 ; \mathrm{k}=201$ ) was positively associated with learning in the TLT. This cluster was identified using a frontal mask, and was corrected for multiple comparisons within the masked volume at $\mathrm{P}<.05$. Pearson's $\mathrm{r}$ values are shown for descriptive purposes only in both panels 

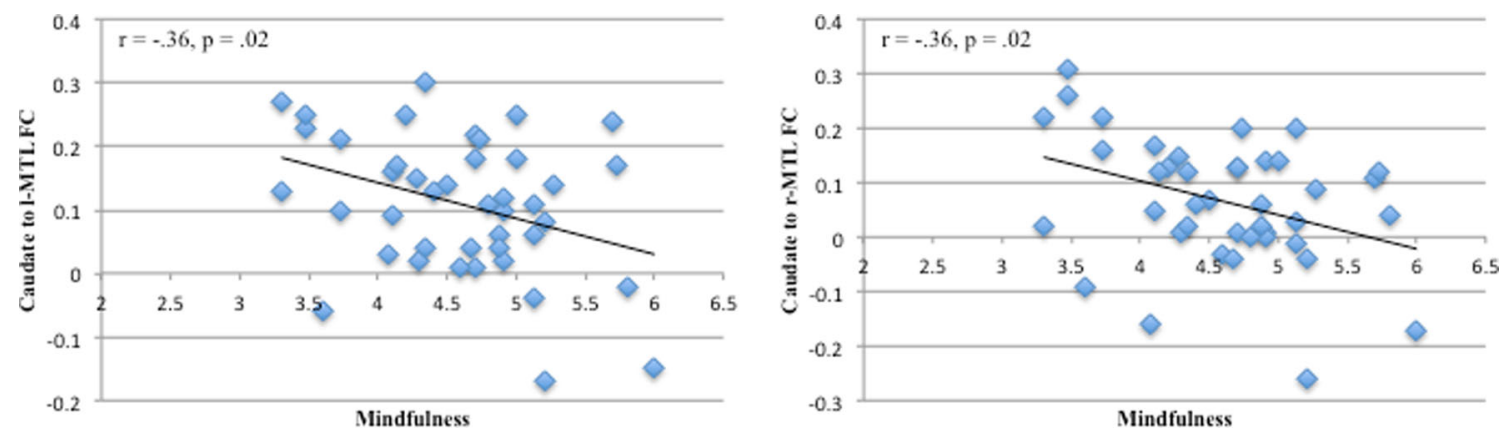

Fig. 4 Task-related functional connectivity of the caudate to the left medial temporal lobe (1-MTL) and right MTL (r-MTL) is also associated with mindfulness in older adults

Each potential mediator was tested in a simple mediation model by calculating bias-corrected $95 \%$ confidence intervals (CIs) using a bootstrapping procedure with 1,000 resamples using the PROCESS macro for SPSS (Hayes, 2013; Preacher \& Hayes, 2008). There was a significant indirect effect of mindfulness on learning through caudate-lMTL connectivity, $\mathrm{b}=-6.0, \mathrm{BCa}$ CI $[-12.68$ to -.62$]$. This represents a mediumto large-sized effect, $\mathrm{K}^{2}=.21, \mathrm{BCa}[.03-.40]$. There was also a significant indirect effect of mindfulness on learning through caudate-rMTL connectivity, $\mathrm{b}=-5.48, \mathrm{BCa} \mathrm{CI}[-11.74$ to $-.20]$, which represents a medium-sized effect, $\mathrm{K}^{2}=.19$, BCa CI [.02-.36].

Figure $5 \mathrm{~b}$ depicts the amount of variance in learning explained by mindfulness alone compared to when caudateIMTL or caudate-rMTL connectivity was included in the model. When mindfulness was the sole predictor of learning, a significant portion of the variance was accounted for $(12 \%)$. However, when either caudate-lMTL or caudate-rMTL was also entered into the model, each was an independently significant predictor, accounting for a significant $38 \%$ and 32.8 $\%$ of the variance in learning, respectively. Further, mindfulness no longer accounted for a significant portion of the variance in learning ( $1.9 \%$ and $2.4 \%$, respectively) following the inclusion of these connections, suggesting that caudateMTL functional connectivity fully mediated the relationship between mindfulness and learning.

To further calculate the degree to which connectivity attenuated the amount of variance in IPSL that can be explained by mindfulness, the amount of variance uniquely associated with mindfulness after removing variance attributable to caudate connectivity to the 1- or r-MTL was subtracted from the amount of variance associated with mindfulness as the sole predictor, and then divided by the amount of variance with mindfulness as the sole predictor (Bennett et al., 2011; Salthouse, 1991). This calculation revealed that connectivity between the caudate and 1- and rMTL attenuated mindfulnessrelated variance in IPSL by $84 \%$ and $80 \%$, respectively.

\section{Discussion}

The present study examined the neural mechanisms underlying the negative relationship between dispositional mindfulness and implicit probabilistic sequence learning (IPSL)

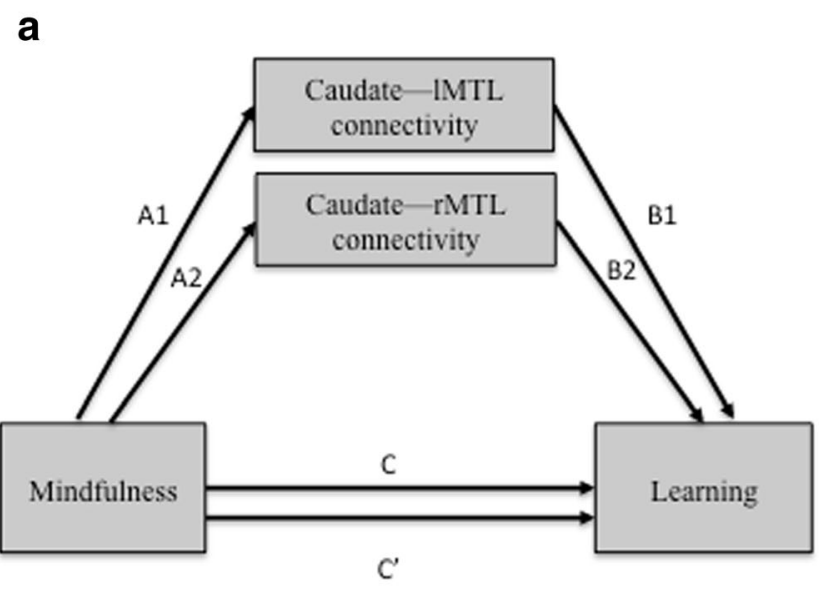

b

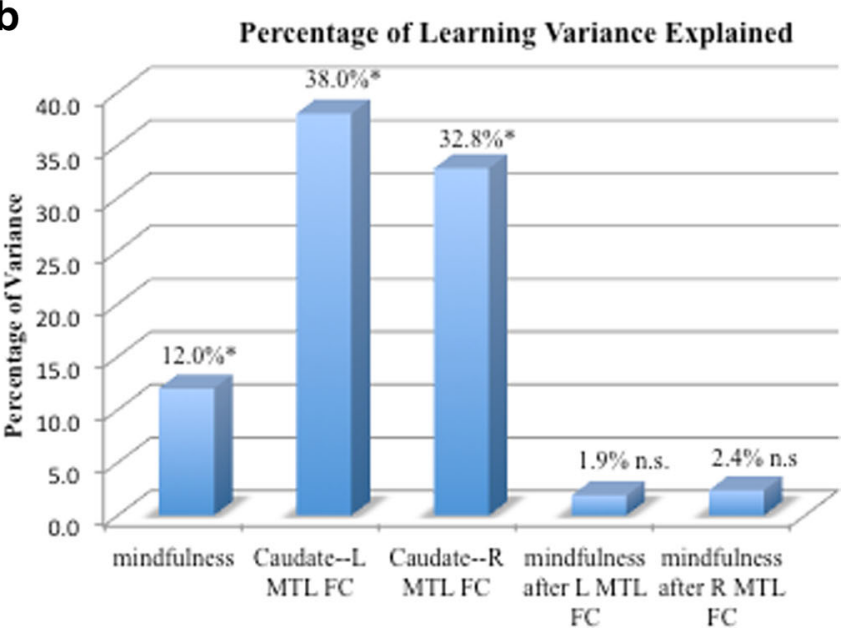

Fig. 5 (a) Conceptual model testing potential mediators of the association between mindfulness and learning. (b) Percentage of variance in learning accounted for by mindfulness before and after caudate-medial temporal lobe (MTL) functional connectivity (FC) is entered into the model 
reported in Stillman et al (2014). Here, at least in our sample of healthy older adults, we replicated the negative relationship between these two behavioral variables. Then, using a seedbased connectivity approach, we identified task-related functional connections associated with individual differences in both learning and mindfulness. The main finding was that caudate-MTL connectivity was positively correlated with IPSL and negatively correlated with mindfulness in this group. Further, the strength of connectivity between these regions mediated the negative relationship between mindfulness and IPSL. Thus, this study is the first to show that the taskrelated functional communication between two learningrelevant regions can account for the relationship between mindfulness and IPSL in a sample of healthy older adults. Discussion of these findings is organized in terms of the three study aims.

\section{Aim 1: Behavioral replication of mindfulness-learning association}

As in Stillman et al. (2014, Study 2) we observed a negative relationship between mindfulness and IPSL in healthy older adults, which could not be attributed to individual differences in age or overall task performance. This represents a replication of the effect in healthy adults and provides further support for the hypothesis that dispositional mindfulness does not benefit and may even impair implicit learning. We also demonstrated that mindfulness was not related to a measure of explicit knowledge in the TLT, indicating that the effect is specific to implicit learning on the task, and not to explicit awareness.

In addition, the present behavioral findings extend our earlier study in several ways. First, we observed the mindfulnessIPSL correlation in a sample of older adults more than double the size used in either of the studies reported in Stillman et al. (2014), thus addressing a major limitation of those studies. In addition, the present sample was more balanced in terms of the gender composition, whereas the samples in our earlier study were mostly female. Finally, the older adults in the present study completed the TLT in the scanner, whereas those in study 2 of our earlier behavioral study completed a different IPSL task (the ASRT) in a behavioral testing room. Thus, these results further support that the relationship between mindfulness and IPSL is not limited to a particular gender, learning task, or testing context.

Nonetheless, two aspects of our behavioral results were unexpected. The first was that older adults did not show evidence of learning at the group level. We usually find that the magnitude of learning is reduced in older compared to younger adults (J.H. Howard Jr. \& Howard, 2013; Rieckmann \& Bäckman, 2009), as it was in the present study. However, we typically do find significant learning at the group level, even for the older adults. The lack of such a group effect in the present study may reflect changes made to the traditional TLT to make it more suitable for the scanner (e.g., fewer trials, jitter in event timing, balanced number of HP vs. LP triplets), but could have made learning more difficult. For example, equating the total number of HP and LP trials, as we did for the first time in this version of the TLT, was different from previous versions of the task in which the position of cue 1 (only) predicted the target location. That is, in the original TLT there was a simple conditional relationship between cue 1 and the target, such that on $80 \%$ of trials the location of cue 1 predicted that the target would appear in a single HP location; the remaining $20 \%$ of the time, cue 1 in the same location was followed by a target in one of the three other possible ("LP") locations. In contrast, in the present version of the TLT, the cue pairs (cue $1+$ cue 2 ) predicted the target location. In other words, here there were 16 conditional probabilities (i.e., the probability of an event given that another has occurred) to learn, whereas there were only four conditional probabilities in the original TLT. In fact, the mean learning scores for both age groups were lower in the present study compared to published work using the original TLT (J.H. Howard Jr. et al., 2008; Simon, Stollstorff, et al., 2011), perhaps accounting for the lack of a group learning effect in the older group.

The second unexpected finding is that we did not replicate the negative correlation between mindfulness and TLT learning in the younger group that we had shown earlier (Stillman et al., 2014, Study 1). The reason for this failure to replicate is unclear, though the changes in the TLT task described above could be important here as well. For example, we saw more evidence of explicit awareness in our younger group's questionnaire responses than we typically see in the TLT, and that group also showed a marginal positive correlation between BDS scores and learning, whereas there was no relationship between the neuropsychological measures and learning in the older group. One possibility for this pattern of results is that the present version of the TLT results in a different balance in neural learning systems, e.g., being more MTL/PFC-driven in the younger group than in the usual TLT. Regardless, our failure to replicate the mindfulness-implicit learning relationship in the young sample indicates that future work is needed to test whether the relationship between dispositional mindfulness and learning holds using different implicit tasks and/or age groups.

\section{Aim 2: Task connectivity related to learning}

Caudate-MTL connectivity Consistent with our prediction, caudate-MTL connectivity was related to IPSL in the TLT in our sample of healthy older adults. Specifically, more positive task-related connectivity between the caudate and clusters in the left and right MTL (at every run) was correlated with higher learning scores. This pattern of results extends those reported in Stillman et al., (2013) in which we demonstrated 
that more connectivity between these regions at rest predicted better subsequent learning in healthy young adults. Because we did not collect fMRI data in that earlier study, however, it was not possible to tell how the caudate and MTL interacted during learning.

The present results are therefore the first to show that more task-related functional connectivity between the caudate and MTL during an IPSL task is beneficial for learning. Since more connectivity between brain regions at the time of measurement is thought to reflect their shared functionality (Fox \& Raichle, 2007), this finding lends further support to a hypothesis put forth in previous fMRI activation studies of IPSL, as well as recent reviews of learning and memory systems, both of which have posited that both the caudate and MTL support learning-related functions during IPSL, despite the fact that this particular type of learning occurs implicitly (Henke, 2010; J.H. Howard Jr. \& Howard, 2013; Schendan et al., 2013; Simon et al., 2012).

An important caveat is that the present study focused on healthy older adults, and so it is possible that the relationship between caudate-MTL connectivity during task and learning would be different in a younger sample. Indeed, there is evidence of functional reorganization of learning systems in older adults, perhaps stemming from the fact that the striatum declines at a faster rate than the MTL during healthy aging (Raz et al., 2005). Furthermore, fMRI studies of IPSL have reported that MTL activation is correlated with better learning at all stages of training for older adults, while it is only correlated with better learning in the early stages of training in younger adults (Rieckmann et al., 2010; Simon et al., 2012). For younger adults, learning in later stages of training is typically correlated with caudate activity, suggesting that MTL activation in late training in older adults may be somehow compensating (albeit not completely successfully) for striatal dysfunction in this age group (Rieckmann et al., 2010; Simon et al., 2012). The finding that caudate-MTL connectivity at all three runs correlated positively with participants' run 3 learning score in this study could therefore reflect a compensatory mechanism by which some older individuals are able to optimize IPSL despite age-related neural changes through the persistent communication between these regions. However, the fact that a similar relationship was found in younger adults in a previous study of resting state connectivity suggests that the positive relationship between caudate-MTL connectivity and learning is not entirely exclusive to older adults (Stillman et al., 2013).

In fact, if stronger caudate-MTL connectivity (as measured here) does support learning across both age groups as we hypothesize, then given that younger adults exhibited more learning that older adults on the TLT in the present study, this group should also exhibit stronger connectivity on average compared to the older adults in this specific connection. Therefore, we compared the strength of older and younger adults' task-related connectivity between the caudate and L- and R-MTL at each run, as well as the cross-run average (Supplementary File 2). We found that younger adults exhibited stronger connectivity than older adults in all cases (all ts $>2.0$, ps <.04). This pattern of results is consistent with our interpretation that stronger caudate-MTL connectivity supports learning. That is, we observed age-group differences in a behavioral measure of learning, as well as in the strength of two connections identified as being learning-related.

Caudate-PFC connectivity Contrary to our predictions, caudate connectivity to a cluster in the right anterior middle/ superior frontal gyrus (BA10) was positively related to learning in older adults. We had initially predicted a negative relationship between learning and caudate-PFC connectivity based on the hypothesis that a prefrontal-based explicit hypothesis testing system competes with a striatal-based implicit procedural system for control of behavior (Filoteo, Lauritzen, \& Maddox, 2010; Poldrack \& Packard, 2003); this hypothesis has been supported by previous behavioral studies of IPSL (Galea et al., 2009; Nemeth et al., 2012).

The findings from the present study, however, suggest that the interplay between these learning systems may be more nuanced than originally thought. For example, there are several distinct frontostriatal loops known to support complex cognitive functions, including implicit ones such as IPSL (Chudasama \& Robbins, 2006). Thus, one possible explanation for the positive relationship between learning and caudate-PFC connectivity we detected is that the nature of the caudate-PFC interactions during IPSL differs by anatomical region. Consistent with this interpretation, the prefrontal cluster we identified (i.e., in the middle/superior frontal gyrus) is located anterior to the dorsolateral prefrontal regions that are the most often implicated in theories of competition (Galea et al., 2009; Halligan \& Oakley, 2012; Nemeth et al., 2012). Rather than supporting regulatory functions per se, this particular region of the PFC is thought to be involved in integrating complex information over time (Prabhakaran, Narayanan, Zhao, \& Gabrieli, 2000). Thus, stronger connectivity between the caudate and this region might enable older individuals to more efficiently integrate the complex probabilistic relationship occurring amongst the sequentially presented cues in the TLT, providing a potential explanation for why task-related connectivity between the caudate and this particular PFC region is positively related to learning success.

\section{Aim 3: Mediation results}

The third goal of this study was to test for potential neural mediators of the mindfulness-IPSL relationship. The caudate-PFC connection identified as being related to learning was not related to mindfulness, and so it did not meet the criteria for mediation (Baron \& Kenny, 1986). However, the two caudate-MTL connections identified as being related to 
learning did qualify as potential mediators, as they were also correlated with mindfulness scores. The results of the (separate) mediation analyses on these connections revealed that both were full mediators of the relationship between mindfulness and IPSL in our older sample, attenuating the amount of variance in IPSL that could be attributed to mindfulness by $84 \%$ and $80 \%$, respectively.

While caudate-MTL communication has been posited to be important to IPSL in previous studies (as discussed above), this is the first time that the communication between these regions has been linked to dispositional mindfulness. It is not clear whether mindfulness alters connectivity between the caudate and MTL systems directly, or by influencing one (or both) systems individually thus indirectly altering their connectivity.

According to a direct account, higher levels of dispositional mindfulness lead to reduced communication between the striatal and MTL learning systems specifically, enabling one (e.g., the MTL system) to more effectively dominate behavior (Chiesa, Calati, \& Serretti, 2011; Chiesa \& Serretti, 2011; McDonald \& Hong, 2013). Since optimal IPSL may depend on efficient interaction/coordination between both the striatal and MTL systems (J.H. Howard Jr. \& Howard, 2013; Reber, 2013; Rieckmann \& Bäckman, 2009), this interpretation would explain why reduced communication between the caudate and MTL leads to lower learning scores; less communication between the two regions might enable the MTL system to dominate throughout the course of IPSL, which would lead to less learning since the striatal system is thought to be optimal in later stages of training (J.H. Howard Jr. \& Howard, 2013; Simon et al., 2012). However, since the older adults did not show a group learning effect by run 3, it's possible that a different pattern of results might have emerged if training was extended.

In contrast, an indirect account suggests that mindfulnessinduced functional and structural changes to these regions (mostly reported in mindfulness training studies) have downstream consequences on their functional communication. In other words, the negative relation between mindfulness and task-related caudate-MTL connectivity in the present sample of older adults could be a byproduct of other changes to these brain regions that are linked to mindfulness, that in turn lead to reduced communication between them. For example, recent fMRI studies have reported that (compared to novices) experienced meditators show less activity in striatal regions during both the anticipation and response phases of reward conditioning tasks (Kirk et al., 2014; Kirk \& Montague, 2015). The meditators' diminished striatal response in these studies is thought to reflect their ability to dampen and/or override anticipatory dopamine responses to reward-related cues, thereby allowing them to act in more controlled, non-reactive, nonhabitual ways (Kirk et al., 2014; Kirk \& Montague, 2015). Unlike the tasks used in these earlier studies, the TLT contains no explicit reward component and is relies on a different striatal subregion (caudate) than that focused on in earlier work (putamen). However, the reduced reactivity of specific striatal regions observed in meditators may result in the entire region becoming more functionally segregated from other brain regions (including the MTL), often leading to a propensity for better explicit functioning, but less learning on tasks, such as IPSL where communication/coordination between these regions is critical. Both of the above accounts are consistent with the mediation model we propose. However, future research is needed to distinguish between them.

\section{Limitations and future directions}

Inferring cause Both of the interpretations we offer above for the mediation results assume that mindfulness is leading to the observed reduction in task-related caudate/MTL connectivity, rather than the reverse. Our ability to make causal inferences regarding the direction of the relationships amongst mindfulness, connectivity, and learning is limited by the correlational nature of our design.

Of course, there is evidence from mindfulness training studies supporting the $a$ priori directionality assumptions we made when conceptualizing the casual pathways in our mediation model (for review see Tang, Hölzel, \& Posner, 2015). Nonetheless, related to the next set of limitations below, there is reason to believe that trained mindfulness and current operationalizations of dispositional mindfulness could be different (Davidson \& Kaszniak, 2015). We did not screen for previous meditation experience in the present study so we cannot examine its relation to implicit learning here. Future work should include implicit learning tasks in neuroimaging studies both before and after mindfulness training in mediation-naïve individuals in order to test whether the mediation model we propose is supported in the training domain as well.

Measuring mindfulness Another set of limitations concerns the complexities in defining and measuring mindfulness. First, we relied on a well established measure (the MAAS), but it is not the only or even necessarily the best measure of dispositional mindfulness. The MAAS is a single-factor scale, while mindfulness is a multifaceted and intangible construct, even for those with years of formal training (Grossman, 2011), and there is still not a single accepted definition of mindfulness in the research community. Critics have argued that the questions comprising scales designed to assess mindfulness, including the MAAS, may instead be measuring closely-related (yet distinct) constructs - -e.g., propensity for mind-wandering, or for everyday lapses in attention. One possibility, for example, is that it is one's propensity for mind wandering (not necessarily the absence of mindfulness) that is linked to implicit learning. 
Second, we used a measure of what is thought to be dispositional mindfulness, i.e., the trait of mindfulness, but we cannot determine for sure whether it is the trait of being mindful or the state of being mindful that is associated with poor implicit learning. These are typically confounded because people who are high in trait mindfulness (as assessed by the MAAS) are, by definition, more likely than those low in mindfulness to actually be in a mindful state at any given time. Thus, it is possible that people high in trait mindfulness have the same capacity for implicit learning as people with low trait mindfulness, but because of their propensity to be in a mindful, they are less apt to use their implicit learning capacity and more apt to rely on their explicit capabilities. These state versus trait explanations could be distinguished by determining whether high MAAS people learn as well as low MAAS people when they are given instructions that encourage them to adopt a non-mindful state during the task.

Chosen seed We chose to use a caudate seed to identify candidate connections for our mediation analyses given the centrality of this region in implicit types of learning. In addition, we had a priori hypotheses regarding how the functional connections of this region might also be modulated by mindfulness. However, the pattern of results we report might differ if other seed regions or approaches to calculating functional connectivity were used. For example, different parts of the basal ganglia and striatum have been associated with explicit vs. implicit learning, and so it's impossible make any overall conclusions about striatal functioning and mindfulness. Future studies could examine these possibilities, as the relationships between functional connectivity, mindfulness, and specific cognitive functions/ behaviors have rarely been investigated and thus remain poorly understood (Tang et al., 2015).

Anatomical connectivity It is notable that task-related functional connectivity between the caudate and MTL relates to both mindfulness and implicit learning in older adults in the present study even though there are few direct anatomical connections between these regions in humans. This is not surprising given evidence that a direct anatomical connection is not necessary for functional connectivity, but it implies there may be other regions mediating their connection (Honey et al., 2009). In the case of implicit sequence learning, for example, prior work using diffusion tensor imaging tractography suggests that DC-MTL functional connectivity may be mediated via the dorsolateral prefrontal cortex (Bennett et al., 2011). Thus, it is possible that the observed connectivity between caudate and MTL was mediated by the DLPFC (though we did not observe relationships consistent with this in the present study), and further, that variability in caudate-DLPFC and
MTL-DLPFC anatomical connections could underlie the wide range of individual differences in learning we observed in the older adult sample. Future studies could combine fMRI functional connectivity and diffusion tensor imaging to examine this possibility.

Psychophysiological interactions versus general task connectivity It should be noted that the present study did not use psychophysiological interaction (PPI) analyses for examining condition-specific changes in connectivity between brain regions during fMRI tasks; we instead collapsed across the HP and LP task conditions for our analyses. Because of this, it is possible that the connections we identified as being related to individual differences in learning (caudate-MTL and caudatePFC) were not supporting learning per se, but rather some other aspect of the task related to learning outcomes. However, psycho-physiological interaction (PPI) analyses are known to have a higher rate of Type II error in eventrelated compared to block-designs (O'Reilly, Woolrich, Behrens, Smith, \& Johansen-Berg, 2012), and so, given our event-related design, we chose to use this alternative approach to decrease the chances of spurious null results. Future fMRI studies could administer IPSL tasks in a blocked design (e.g., pattern vs. random blocks) in order to more effectively implement PPI analyses.

\section{Conclusions}

The present results implicate functional connectivity between the caudate and MTL as a neural mechanism underlying the previously reported behavioral relationship between dispositional mindfulness and IPSL (Stillman et al., 2014) for healthy older adults. These findings contribute to understanding how specific traits (i.e., mindfulness) might influence aptitude for basic cognitive functions, such as acquiring complex regularities without awareness or intent. They also highlight the need for more studies examining the complex interplay between mindfulness (including one's natural propensity for it), the brain, and behavior.

Acknowledgments Special thanks to Jessica Simon, Alyssa Coffin, and Priya Santhanam for developing the version of the TLT used in the present study. The authors are also very grateful to Halley Feldman and Eileen Rasmussen for coordinating study recruitment and testing. We would also like to thank Russell Marchese, Derek Embry, and Erica Rabinovich for assistance with data collection, as well as R. Scott Turner, John VanMeter, Howard Aizenstein, David Madden, and Anna Greenwald for technical assistance and training. This research was supported by NIA/NIH grants RO1AG036863 to D.V.H. and J.H.H., Jr. and F31AG047037 to CMS. 


\section{Appendix}

Table 2 List of all possible triplets in the TLT

Triplets presented 331

during TLT

113

124

233

241

334

342

411

422

112

114

122

123

132

133

134

142

143

144

211

213

214

221

223

224

231

234

244

243

311

312

314

321

322

324

331

332

341

344

412

413

421

423

431

432

433
Table 2 (continued)

\section{1}

442

443

TLT Triplets Learning Task

\section{References}

Aizenstein, H. J., Butters, M. A., Clark, K. A., Figurski, J. L., Andrew Stenger, V., Nebes, R. D., ... Carter, C. S. (2006). Prefrontal and striatal activation in elderly subjects during concurrent implicit and explicit sequence learning. Neurobiology of Aging, 27(5), 741-751. doi:10.1016/j.neurobiolaging.2005.03.017

Albouy, G., Sterpenich, V., Balteau, E., Vandewalle, G., Desseilles, M., Dang-Vu, T., ... Maquet, P. (2008). Both the hippocampus and striatum are involved in consolidation of motor sequence memory. Neuron, 58(2), 261-272. doi:10.1016/j.neuron.2008.02.008

Baron, R. M., \& Kenny, D. A. (1986). The moderator-mediator variable distinction in social psychological research: Conceptual, strategic, and statistical considerations. Journal of Personality and Social Psychology, 51(6), 1173-1182.

Bennett, I. J., Madden, D. J., Vaidya, C. J., Howard, J. H., Jr., \& Howard, D. V. (2011). White matter integrity correlates of implicit sequence learning in healthy aging. Neurobiology of Aging, 32(12), 2317.e112. doi:10.1016/j.neurobiolaging.2010.03.017

Brown, K. W., \& Ryan, R. M. (2003). The benefits of being present: Mindfulness and its role in psychological well-being. Journal of Personality and Social Psychology, 84(4), 822-848. doi:10.1037/ 0022-3514.84.4.822

Carlson, L. E., \& Brown, K. W. (2005). Validation of the Mindful Attention Awareness Scale in a cancer population. Journal of Psychosomatic Research, 58(1), 29-33. doi:10.1016/j.jpsychores. 2004.04.366

Chiesa, A., Calati, R., \& Serretti, A. (2011). Does mindfulness training improve cognitive abilities? A systematic review of neuropsychological findings. Clinical Psychology Review, 31(3), 449-464. doi: 10.1016/j.cpr.2010.11.003

Chiesa, A., \& Serretti, A. (2011). Mindfulness based cognitive therapy for psychiatric disorders: A systematic review and meta-analysis. Psychiatry Research, 187(3), 441-453. doi:10.1016/j.psychres. 2010.08.011

Chiesa, A., Serretti, A., \& Jakobsen, J. C. (2013). Mindfulness: Topdown or bottom-up emotion regulation strategy? Clinical Psychology Review, 33(1), 82-96. doi:10.1016/j.cpr.2012.10.006

Chudasama, Y., \& Robbins, T. W. (2006). Functions of frontostriatal systems in cognition: Comparative neuropsychopharmacological studies in rats, monkeys and humans. Biological Psychology, 73(1), 19-38. doi:10.1016/j.biopsycho.2006.01.005

Cohen, A., \& Curran, T. (1993). On tasks, knowledge, correlations, and dissociations: Comment on Perruchet and Amorim (1992). Journal of Experimental Psychology: Learning, Memory, and Cognition, 19(6), 1431-1437. doi:10.1037/0278-7393.19.6.1431

Cox, R. W. (1996). AFNI: software for analysis and visualization of functional magnetic resonance neuroimages. Computers and Biomedical Research, an International Journal, 29(3), 162-173.

Davidson, R. J., \& Kaszniak, A. W. (2015). Conceptual and methodological issues in research on mindfulness and meditation. American Psychologist, 70(7), 581-592. doi:10.1037/a0039512

Filoteo, J. V., Lauritzen, S., \& Maddox, W. T. (2010). Removing the frontal lobes: The effects of engaging executive functions on perceptual category learning. Psychological Science, 21(3), 415-423. doi:10.1177/0956797610362646 
Fox, M. D., \& Raichle, M. E. (2007). Spontaneous fluctuations in brain activity observed with functional magnetic resonance imaging. Nature Reviews Neuroscience, 8(9), 700-711. doi:10.1038/nrn2201

Galea, J. M., Albert, N. B., Ditye, T., \& Miall, R. C. (2009). Disruption of the dorsolateral prefrontal cortex facilitates the consolidation of procedural skills. Journal of Cognitive Neuroscience, 22(6), 11581164. doi:10.1162/jocn.2009.21259

Gamble, K. R., Cummings Jr., T. J., Lo, S. E., Ghosh, P. T., Howard Jr., J. H., \& Howard, D. V. (2014). Implicit sequence learning in people with Parkinson's disease. Frontiers in Human Neuroscience, 8, 563. doi:10.3389/fnhum.2014.00563

Garland, E., Froeliger, B., \& Howard, M. (2014). Mindfulness training targets neurocognitive mechanisms of addiction at the attentionappraisal-emotion interface. Addictive Disorders and Behavioral Dyscontrol, 4, 173. doi:10.3389/fpsyt.2013.00173

Gheysen, F., Van Opstal, F., Roggeman, C., Van Waelvelde, H., \& Fias, W. (2011). The neural basis of implicit perceptual sequence learning. Frontiers in Human Neuroscience, 5, 1-12. doi:10.3389/fnhum. 2011.00137

Grant, J. A., Courtemanche, J., Duerden, E. G., Duncan, G. H., \& Rainville, P. (2010). Cortical thickness and pain sensitivity in zen meditators. Emotion, 10(1), 43-53. doi:10.1037/a0018334

Grossman, P. (2011). Defining mindfulness by how poorly I think I pay attention during everyday awareness and other intractable problems for psychology's (re)invention of mindfulness: Comment on Brown et al. Psychological Assessment, 23(4), 1034-1040. doi:10.1037/ a0022713

Halligan, P. W., \& Oakley, D. A. (2012). Hypnosis and cognitive neuroscience: Bridging the gap. Cortex; a Journal Devoted to the Study of the Nervous System and Behavior. doi:10.1016/j.cortex.2012.12. 002

Hayes, A. F. (2013). Introduction to Mediation, Moderation, and Conditional Process Analysis: A Regression-Based Approach. New York: Guilford Press.

Henke, K. (2010). A model for memory systems based on processing modes rather than consciousness. Nature Reviews Neuroscience, 11(7), 523-532. doi:10.1038/nrn2850

Holzel, B. K., Carmody, J., Vangel, M., Congleton, C., Yerramsetti, S. M., Gard, T., \& Lazar, S. W. (2011). Mindfulness practice leads to increases in regional brain gray matter density. Psychiatry Research, 191(1), 36-43. doi:10.1016/j.pscychresns.2010.08.006

Honey, C. J., Sporns, O., Cammoun, L., Gigandet, X., Thiran, J. P., Meuli, R., \& Hagmann, P. (2009). Predicting human resting-state functional connectivity from structural connectivity. Proceedings of the National Academy of Sciences of the United States of America, 106(6), 2035-2040. doi:10.1073/pnas.0811168106

Howard, J. H., Jr, \& Howard, D. V. (2013). Aging mind and brain: is implicit learning spared in healthy aging? Frontiers in Psychology, 4, 817. doi:10.3389/fpsyg.2013.00817

Howard, J. H., Jr., Howard, D. V., Dennis, N. A., \& Kelly, A. J. (2008). Implicit learning of predictive relationships in three-element visual sequences by young and old adults. Journal of Experimental Psychology: Learning, Memory, and Cognition, 34(5), 1139-1157. doi: $10.1037 / \mathrm{a} 0012797$

Howard, D. V., Howard, J. H., Japikse, K., DiYanni, C., Thompson, A., \& Somberg, R. (2004). Implicit Sequence Learning: Effects of Level of Structure, Adult Age, and Extended Practice. Psychology and Aging, 19(1), 79-92. doi:10.1037/0882-7974.19.1.79

Jha, A. P., Stanley, E. A., Kiyonaga, A., Wong, L., \& Gelfand, L. (2010). Examining the protective effects of mindfulness training on working memory capacity and affective experience. Emotion, 10(1), 54-64. doi:10.1037/a0018438

Kirk, U., Brown, K. W., \& Downar, J. (2014). Adaptive neural reward processing during anticipation and receipt of monetary rewards in mindfulness meditators. Social Cognitive and Affective Neuroscience, nsu112. doi:10.1093/scan/nsu112
Kirk, U., \& Montague, P. R. (2015). Mindfulness meditation modulates reward prediction errors in a passive conditioning task. Cognition, 6 , 90. doi:10.3389/fpsyg. 2015.00090

Luders, E., Toga, A. W., Lepore, N., \& Gaser, C. (2009). The underlying anatomical correlates of long-term meditation: Larger hippocampal and frontal volumes of gray matter. NeuroImage, 45(3), 672-678. doi:10.1016/j.neuroimage.2011.05.075

Lutz, J., Herwig, U., Opialla, S., Hittmeyer, A., Jäncke, L., Rufer, M., ... Brühl, A. B. (2014). Mindfulness and emotion regulation - an fMRI study. Social Cognitive and Affective Neuroscience, 9(6), 776-785. doi:10.1093/scan/nst043

Maldjian, J. A., Laurienti, P. J., Kraft, R. A., \& Burdette, J. H. (2003). An automated method for neuroanatomic and cytoarchitectonic atlasbased interrogation of fMRI data sets. NeuroImage, 19(3), 12331239. doi:10.1016/S1053-8119(03)00169-1

Maldjian, J. A., Laurienti, P. J., \& Burdette, J. H. (2004). Precentral gyrus discrepancy in electronic versions of the Talairach atlas. NeuroImage, 21(1), 450-455. doi:10.1016/j.neuroimage.2003.09. 032

McDonald, R. J., \& Hong, N. S. (2013). How does a specific learning and memory system in the mammalian brain gain control of behavior?: Memory Systems and Behavioral Control. Hippocampus, 23(11), 1084-1102. doi:10.1002/hipo.22177

Mrazek, M. D., Franklin, M. S., Phillips, D. T., Baird, B., \& Schooler, J. W. (2013). Mindfulness training improves working memory capacity and GRE performance while reducing mind wandering. Psychological Science, 24(5), 776-781. doi:10.1177/ 0956797612459659

Mrazek, M. D., Smallwood, J., \& Schooler, J. W. (2012). Mindfulness and mind-wandering: Finding convergence through opposing constructs. Emotion, 12(3), 442-448. doi:10.1037/a0026678

Nemeth, D., Janacsek, K., Polner, B., \& Kovacs, Z. A. (2012). Boosting Human Learning by Hypnosis. Cerebral Cortex, 23(4), 801-805. doi:10.1093/cercor/bhs068

O’Reilly, J. X., Woolrich, M. W., Behrens, T. E. J., Smith, S. M., \& Johansen-Berg, H. (2012). Tools of the trade: psychophysiological interactions and functional connectivity. Social Cognitive and Affective Neuroscience, 7(5), 604-609. doi:10.1093/scan/nss055

Perruchet, P., \& Amorim, M.-A. (1992). Conscious knowledge and changes in performance in sequence learning: Evidence against dissociation. Journal of Experimental Psychology: Learning, Memory, and Cognition, 18(4), 785-800. doi:10.1037/0278-7393.18.4.785

Perruchet, P., \& Gallego, J. (1993). Association between conscious knowledge and performance in normal subjects: Reply to Cohen and Curran (1993) and Willingham, Greeley, and Bardone (1993). Journal of Experimental Psychology: Learning, Memory, and Cognition, 19(6), 1438-1444. doi:10.1037/0278-7393.19.6.1438

Pickut, B. A., Van Hecke, W., Kerckhofs, E., Mariën, P., Vanneste, S., Cras, P., \& Parizel, P. M. (2013). Mindfulness based intervention in Parkinson's disease leads to structural brain changes on MRI: A randomized controlled longitudinal trial. Clinical Neurology and Neurosurgery, 115(12), 2419-2425. doi:10.1016/j.clineuro.2013. 10.002

Poldrack, R. A., Clark, J., Paré-Blagoev, E. J., Shohamy, D., Creso Moyano, J., Myers, C., \& Gluck, M. A. (2001). Interactive memory systems in the human brain. Nature, 414(6863), 546-550. doi:10. 1038/35107080

Poldrack, R. A., \& Packard, M. G. (2003). Competition among multiple memory systems: Converging evidence from animal and human brain studies. Neuropsychologia, 41(3), 245-251. doi:10.1016/ S0028-3932(02)00157-4

Power, J. D., Barnes, K. A., Snyder, A. Z., Schlaggar, B. L., \& Petersen, S. E. (2012). Spurious but systematic correlations in functional connectivity MRI networks arise from subject motion. NeuroImage, 59(3), 2142-2154. doi:10.1016/j.neuroimage.2011.10.018 
Prabhakaran, V., Narayanan, K., Zhao, Z., \& Gabrieli, J. D. E. (2000). Integration of diverse information in working memory within the frontal lobe. Nature Neuroscience, 3(1), 85-90. doi:10.1038/71156

Preacher, K. J., \& Hayes, A. F. (2008). Asymptotic and resampling strategies for assessing and comparing indirect effects in multiple mediator models. Behavior Research Methods, 40(3), 879-891. doi:10. 3758/BRM.40.3.879

Raichle, M. E. (2010). Two views of brain function. Trends in Cognitive Sciences, 14(4), 180-190. doi:10.1016/j.tics.2010.01.008

Raz, N., Lindenberger, U., Rodrigue, K. M., Kennedy, K. M., Head, D., Williamson, A., ... Acker, J. D. (2005). Regional Brain Changes in Aging Healthy Adults: General Trends, Individual Differences and Modifiers. Cerebral Cortex, 15(11), 1676-1689. doi:10.1093/ cercor/bhi044

Reber, P. J. (2013). The neural basis of implicit learning and memory: A review of neuropsychological and neuroimaging research. Neuropsychologia, 51(10), 2026-2042. doi:10.1016/j. neuropsychologia.2013.06.019

Rieckmann, A., \& Bäckman, L. (2009). Implicit learning in aging: Extant patterns and new directions. Neuropsychology Review, 19(4), 490503. doi:10.1007/s11065-009-9117-y

Rieckmann, A., Fischer, H., \& Bäckman, L. (2010). Activation in striatum and medial temporal lobe during sequence learning in younger and older adults: Relations to performance. NeuroImage, 50(3), 1303-1312. doi:10.1016/j.neuroimage.2010.01.015

Rose, M., Haider, H., Weiller, C., \& Büchel, C. (2002). The role of medial temporal lobe structures in implicit learning: An event-related fMRI study. Neuron, 36(6), 1221-1231. doi:10.1016/j.bbr.2011.03.031

Saad, Z. S., Gotts, S. J., Murphy, K., Chen, G., Jo, H. J., Martin, A., \& Cox, R. W. (2012). Trouble at rest: How correlation patterns and group differences become distorted after global signal regression. Brain Connectivity, 2(1), 25-32. doi:10.1089/brain.2012.0080

Salthouse, T. A. (1991). Mediation of Adult Age Differences in Cognition by Reductions in Working Memory and Speed of Processing. Psychological Science, 2(3), 179-183. doi:10.1111/j.1467-9280. 1991.tb00127.x

Schendan, H. E., Searl, M. M., Melrose, R. J., \& Stern, C. E. (2003). An fMRI Study of the Role of the Medial Temporal Lobe in Implicit and Explicit Sequence Learning. Neuron, 37(6), 1013-1025. doi:10. 1016/S0896-6273(03)00123-5

Schendan, H. E., Tinaz, S., Maher, S. M., \& Stern, C. E. (2013). Frontostriatal and mediotemporal lobe contributions to implicit higher-order spatial sequence learning declines in aging and Parkinson's disease. Behavioral Neuroscience, 127(2), 204-221. doi:10.1037/a0032012

Schmertz, S. K., Anderson, P. L., \& Robins, D. L. (2009). The relation between self-report mindfulness and performance on tasks of sustained attention. Journal of Psychopathology and Behavioral Assessment, 31(1), 60-66. doi:10.1007/s10862-008-9086-0

Shapiro, S. L., Carlson, L. E., Astin, J. A., \& Freedman, B. (2006). Mechanisms of mindfulness. Journal of Clinical Psychology, 62(3), 373-386. doi:10.1002/jclp.20237

Shaurya Prakash, R., De Leon, A. A., Klatt, M., Malarkey, W., \& Patterson, B. (2012). Mindfulness disposition and default-mode network connectivity in older adults. Social Cognitive and Affective Neuroscience, 8(1), 112-117. doi:10.1093/scan/nss115

Simon, J. R., Howard, J. H., Jr., \& Howard, D. V. (2011a). Age differences in implicit learning of probabilistic unstructured sequences. The Journals of Gerontology Series B, Psychological Sciences and Social Sciences, 66(1), 32-38. doi:10.1093/geronb/gbq066

Simon, J. R., Stollstorff, M., Westbay, L. C., Vaidya, C. J., Howard, J. H., Jr., \& Howard, D. V. (2011b). Dopamine transporter genotype predicts implicit sequence learning. Behavioural Brain Research, 216(1), 452-457. doi:10.1016/j.bbr.2010.08.043

Simon, J. R., Vaidya, C. J., Howard, J. H., Jr., \& Howard, D. V. (2012). The effects of aging on the neural basis of implicit associative learning in a probabilistic triplets learning task. Journal of Cognitive Neuroscience, 24(2), 451-463. doi:10.1162/jocn_a 00116

Soetens, E., Melis, A., \& Notebaert, W. (2004). Sequence learning and sequential effects. Psychological Research, 69(1-2), 124-137. doi: 10.1007/s00426-003-0163-4

Squire, L. R. (2004). Memory systems of the brain: A brief history and current perspective. Neurobiology of Learning and Memory, 82(3), 171-177. doi:10.1016/j.nlm.2004.06.005

Stillman, C. M., Feldman, H., Wambach, C. G., Howard, J. H., \& Howard, D. V. (2014). Dispositional mindfulness is associated with reduced implicit learning. Consciousness and Cognition, 28, 141150. doi:10.1016/j.concog.2014.07.002

Stillman, C. M., Gordon, E. M., Simon, J. R., Vaidya, C. J., Howard, D. V., \& Howard, J. H. (2013). Caudate Resting Connectivity Predicts Implicit Probabilistic Sequence Learning. Brain Connectivity, 3(6), 601-610. doi:10.1089/brain.2013.0169

Tang, Y.-Y., Hölzel, B. K., \& Posner, M. I. (2015). The neuroscience of mindfulness meditation. Nature Reviews Neuroscience, 16(4), 213 225. doi:10.1038/nrn3916

Tang, Y.-Y., Lu, Q., Geng, X., Stein, E. A., Yang, Y., \& Posner, M. I. (2010). Short-term meditation induces white matter changes in the anterior cingulate. Proceedings of the National Academy of Sciences of the United States of America, 107(35), 15649-15652. doi:10. 1073/pnas.1011043107

Tang, Y.-Y., Ma, Y., Fan, Y., Feng, H., Wang, J., Feng, S., ... Fan, M. (2009). Central and autonomic nervous system interaction is altered by short-term meditation. Proceedings of the National Academy of Sciences of the United States of America, 106(22), 8865-8870. doi:10.1073/pnas.0904031106

Tang, Y.-Y., \& Posner, M. I. (2012). Special issue on mindfulness neuroscience. Social Cognitive and Affective Neuroscience, 8, 1-3. doi: $10.1093 /$ scan $/$ nss 104

Tang, Y.-Y., Tang, R., \& Posner, M. I. (2013). Brief meditation training induces smoking reduction. Proceedings of the National Academy of Sciences, 10, 13971-13975. doi:10.1073/pnas.1311887110

Taren, A. A., Creswell, J. D., \& Gianaros, P. J. (2013). Dispositional mindfulness co-varies with smaller amygdala and caudate volumes in community adults. PLOS ONE, 8, e64574. doi:10.1371/journal. pone. 0064574

Tzourio-Mazoyer, N., Landeau, B., Papathanassiou, D., Crivello, F., Etard, O., Delcroix, N., ... Joliot, M. (2002). Automated anatomical labeling of activations in SPM using a macroscopic anatomical parcellation of the MNI MRI single-subject brain. NeuroImage, 15(1), 273-289. doi:10.1006/nimg.2001.0978

Vago, D. R. P. D., \& David, S. A. M. D. (2012). Self-awareness, selfregulation, and self-transcendence (S-ART): A framework for understanding the neurobiological mechanisms of mindfulness. Frontiers in Human Neuroscience, 6, 296. doi:10.3389/fnhum. 2012.00296

Van-Dijk, K. R. A., Hedden, T., Venkataraman, A., Evans, K. C., Lazar, S. W., \& Buckner, R. L. (2010). Intrinsic functional connectivity ss a tool for human connectomics: Theory, properties, and optimization. Journal of Neurophysiology, 103(1), 297-321. doi:10.1152/jn. 00783.2009

Ward, B. D. (2000). Simultaneous inference for FMRI data. AFNI AlphaSim Documentation, Medical College of Wisconsin

Westbrook, C., Creswell, J. D., Tabibnia, G., Julson, E., Kober, H., \& Tindle, H. A. (2013). Mindful attention reduces neural and selfreported cue-induced craving in smokers. Social Cognitive and Affective Neuroscience, 8(1), 73-84. doi:10.1093/scan/nsr076

Whitmarsh, S., Uddén, J., Barendregt, H., \& Petersson, K. M. (2013). Mindfulness reduces habitual responding based on implicit knowledge: Evidence from artificial grammar learning. Consciousness and Cognition, 22(3), 833-845. doi:10.1016/j.concog.2013.05.007 
Willingham, D. B., Greeley, T., \& Bardone, A. M. (1993). Dissociation in a serial response time task using a recognition measure: Comment on Perruchet and Amorim (1992). Journal of Experimental Psychology: Learning, Memory, and Cognition, 19(6), 14241430. doi:10.1037/0278-7393.19.6.1424
Zeidan, F., Martucci, K. T., Kraft, R. A., Gordon, N. S., McHaffie, J. G., \& Coghill, R. C. (2011). Brain Mechanisms Supporting the Modulation of Pain by Mindfulness Meditation. The Journal of Neuroscience, 31(14), 5540-5548. doi:10.1523/JNEUROSCI. 5791-10.2011 